\title{
BUCHBESPRECHUNGEN
}

\author{
R. C. Hingorani
}

\section{Modern International Law}

Oceana Publications, Inc., Dobbs Ferry, New York, 1979, XII, 437 S.

Völkerrechtliche Lehrbücher oder Gesamtdarstellungen von prominenten Rechtslehrern aus Staaten außerhalb Europas und Amerikas erscheinen in den letzten Jahren immer häufiger. Die neuesten Arbeiten des Japaners Takano und des Nigerianers Elias sind kürzlich in dieser Zeitschrift vorgestellt worden ${ }^{1}$. Nunmehr ist über das Lehrbuch des Inders Hingorani zu berichten, der - anders als die beiden genannten Autoren und anders auch als Inder, die in früheren Jahren völkerrechtliche Lehrbücher vorgelegt haben ${ }^{2}-$, das Ziel verfolgt, die Sicht der afroasiatischen Staaten zu Einzelproblemen des Völkerrechts zu verdeutlichen, und dies für den auswärtigen Interessierten ebenso wie für den akademischen Unterricht in Asien und Afrika selbst, der bislang weitgehend auf ,,fremde“ Lehrdarstellungen angewiesen ist. Hingoranis Vorwort formuliert unmißverständlich, es sei das Anliegen des Buches, ,, to serve the sole purpose of reflecting Afro-Asian views on various aspects of international law".

Der indische Völkerrechtler widmet sich damit einer Aufgabe, die schon lange der Erfüllung harrt. Die Art und Weise, wie er sie löst, verrät viel über den gegenwärtigen Stand der Völkerrechtsentwicklung und verdoutlicht, wie verfehlt es ist, schematisch ,,westliches“ und ,,neues"Völkerrecht gegeneinander ausspielen zu wollen: Vielmehr ist unter den gegebenen Umständen die Suche nach einer Modifizierung tradierten Rechts geboten, unter sorgfältiger Prüfung, welche der überkommenen Regeln bewahrt zu werden verdienen. So versucht Hingorani, ausgehend von herkömmlicher Systematik und Begrifflichkeit, von altvertrauten leading cases der Völkerrechtswissenschaft (nicht nur Trail Smelter und Caroline, auch der Clipperton Island und der Eastern Greenland Case erscheinen) kritisch zu fragen „,Cui bono?" und - wo ihm dies politisch sinnvoll erscheint - Gegenpositionen de iure lata oder ferenda zu entwickeln. Dabei argumentiert er zumeist erfreulich offen und kompetent, verschleiert nicht die bestehenden Kontroversen. Mehr auf der Darstellung von ,claims“, , aspirations“, ,tendencies“, als auf solcher etablierter Normen liegt das Schwergewicht. Wichtig in diesem Zusammenhang ist etwa die Position Hingoranis zur Bindung ,,neuer“" Staaten an Völkergewohnheitsrecht, das ohne ihre Mitwirkung entstanden ist. Der Autor betont hier daš Konsensprinzip, verneint jed wede Bindung, die nicht ausdrücklich oder stillschweigend, jedenfalls aber durch einen identifizierbaren Akt des einzelnen Staates zustande gekommen ist. Zutreffenderweise (die überwiegende Auffassung im europäischen Schrifttum sieht dies freilich nach wie vor anders) wird herausgearbeitet, daß jede andere Beurteilung mit der Struktur der gegenwärtigen Völkerrechtsordnung nicht zu vereinen ist (S. 14); nicht Stellung genommen allerdings wird zu der Frage, warum nur ehemalige Kolonien das Privileg haben sollen, im Wege des ,,pick and choose“ die ihnen genehmen Gewohnheitsrechtsregeln zu akzeptieren, nicht aber Staaten, die zwar formell seit langem der Volkerrechtsgemeinschaft angehören, aber - etwa nach einem revolutionären Umsturz - in völlig neuer Rolle Mitglieder dieser Gemeinschaft sind (man denke an den Iran). Wenn es eine unzumutbare Härte für die Bevölkerungen der ehemaligen Kolonien sein soll, in ,,formales“

1 VRU 13 (1980), S 274 bzw. S. 185.

2 Vgl. etwa Mahajan, Public International Law, 5. Aufl. 1974. 
Völkerrecht ,,hineingeboren“ zu werden (um das Bild O’Connels zu verwenden), dann könnte dies auch beim Úbergang von einer Regierung zur anderen gelten.

Der Rekurs auf Naturrecht, zeitweilig von vielen Vertretern der Dritten Welt geschmäht als typisches Instrument klassischer westlicher Jurisprudenz, die die Werte christlich-europäischer Tradition sogar den internationalen Beziehungen aufstülpen möchte, begegnet auch bei Hingorani (S. 18, S. 223). Erneut zeigt sich hier, wie nicht nur einzelne völkerrechtliche Normen, sondern auch Grundkonzepte in den Dienst aufs Ergebnis schauender Argumentation gestellt werden - ein Vorgang, der, wenn er offen erfolgt, der völkerrechtlichen Diskussion nur nützen kann.

Die eigenen Wege, die Hingorani im Aufbau seiner Lehrdarstellung geht, mögen gleichfalls von dem Bestreben veranlaßt sein, Dritte-Welt-Positionen hervorzuheben, nur scheint dies nicht durchweg gelungen. Eine anregende Idee ist es sicherlich, das gesamte Seerecht als Teil der ,,General Principles“ auszuweisen; nicht nur die gegenwärtige und künftige Bedeutung des Seerechts wird auf diese Weise dokumentiert, auch die Verwobenheit vieler Entwicklungen der siebziger Jahre mit allgemeinen Völkerrechtsfragen wird hervorgehoben. Daß aber der völkerrechtliche Vertrag in einem eigenen Kapitel weit entfernt von dem Kapitel über Rechtsquellen,.daß ,Intervention“ unter „Disputes“ behandelt, schließlich der systematische Aufbau zerrissen wird durch einen Abschnitt ,,Recent Trends“, dessen Inhalt an anderen Stellen des Buches hätten integriert werden können, erscheint wenig überzeugend.

Kritisch anzumerken ist auch, daß der Autor seinen Anspruch, für die gesamte Dritte Welt zu sprechen (abgesehen von der schon zitierten Vorbemerkung, wird dies auch durch eine Dedizierung an ,,Third World Countries“ zum Ausdruck gebracht), mitunter zu vergessen scheint. Wäre es sonst möglich, daß sich die Darstellung des internationalen Enteignungsrechts hauptsächlich an indischen Beispielen orientiert (Chile wird nicht erwähnt), daß das Verhältnis des innerstaatlichen Rechts zum Völkerrecht anhand der Beispiele Vereinigtes Königreich, Vereinigte Staaten, Indien erläutert wird, daß die Ausführungen zur Weltinformationsordnung die Initiativen der UNESCO vernachlässigen, diejenigen zur ,,Intervention auf Ersuchen" (besonders wichtig in Asien und Afrika: Man denke an die Rolle Kubas, Frankreichs, der Vereinigten Staaten in Afrika, der Sowjetunion in Afghanistan) sich einer klaren Stellungnahme enthalten?

Was Hingorani schließlich zum Verhältnis Bhutans zu Indien zu sagen hat, könnte sich mutatis mutandis auch in einem Lehrbuch aus ,,kolonialer“ Denkschule finden: Der Begriff Protektorat, geprägt im Zeitalter des Imperialismus, wird diskussionslos übernommen, der Freundschaftsvertrag zwischen Indien und Bhutan von 1949, dessen Artikel 2 Gegenstand heftiger politischer und juristischer Kontroversen gewesen is $\mathrm{t}^{3}$, verfälschend paraphrasiert: Seine Nichteinmischungsklausel wird nicht erwähnt, statt ,Bhutan agrees to be guided by the advice of the Government of India" ${ }^{4}$ heißt es, der Vertrag berechtige Indien, ,to govern Bhutan's external relations “ (S. 36) - gerade das ist aber nur die offizielle indische Interpretation.

Hingoranis Werk ist - ungeachtet seiner Schwächen - eine wichtige Neuerscheinung. Abgesehen von den angeführten Punkten wird es seinem Anspruch, ein eigenständiges modernes Völkerrechtslehrbuch zu sein, das die Rolle der Staaten Afrikas und Asiens in den Mittelpunkt rückt, weitgehend gerecht. Der Stil des Autors ist unkompliziert, grenzt mitunter ans Spaßige; die Kapitel sind in sich manchmal fast lexikonartig gegliedert, so daß wichtige Be-

\footnotetext{
3 Vgl. dazu meine Besprechung des Buches von Rose, The Politics of Bhutan, 1977, mit Nachweisen aus jüngster Zeit, VRU 13 (1980), S. 171 .

4 Text des Vertrages bei Hecker, Sikkim und Bhutan, 1970, S. 61.
} 
griffe leicht aufgefunden werden können. Es ist zu hoffen, daß Völkerrechtswissenschaft und Lehrbetrieb die vielfältigen Gelegenheiten zur Anregung und Diskussion nutzen werden, die ihnen dieses Buch bietet. Der Autor selbst sollte in einer Neuauflage stärker die Praxis und die Auffassungen von anderen Staaten der Dritten Welt als Indien berücksichtigen und einige Probleme eindringlicher behandeln (die Staatenverantwortlichkeit, die Bedeutung von Resolutionen der Generalversammlung der Vereinten Nationen). Die Chance, ein neues Standardwerk zu etablieren, scheint gegeben.

Philip Kunig

\section{Muhammed Massarrat}

\section{Weltenergieproduktion und Neuordnung der Weltwirtschaft}

Die Weltarbeitsteilung und die Neuverteilung des Reichtums in der Welt Campus Verlag, Frankfurt - New York, 1980, 294 S.

Solange die Versorgung des Industriestaaten mit billiger Energie gewährleistet und damit aus ihrer Sicht - problemlos war, blieb die Untersuchung der Bedingungen der Produktion und Vermarktung von Energie einigen wenigen Experten vorbehalten, die für ihre Arbeiten kaum mit einem großen Publikum rechnen konnten.

Spätestens jedoch mit der explosionsartigen Erhöhung des Ölpreises von 1973/74 änderte sich das Bild: die ,Energiekrise“ ist in aller Munde, und neo-malthusianische Knappheitsvisionen haben den bislang vorherrschenden Wachstumsoptimismus gründlich verdüstert. Es kann daher kaum überraschen, daß Untersuchungen zum Energieproblem seither Konjunktur haben.

Es ist ein Verdienst Masserrats, daß es ihm gelungen ist, die gesellschaftlichen Bedingungen der Knappheit natürlicher Ressourcen herausgearbeitet und damit die gängigen Knappheitsvorstellungen, wie sie nicht zuletzt durch die Arbeiten des „Club of Rome“ popularisiert wurden, relativiert zu haben. Ausgangspunkt seiner Analyse ist die Rententheorie, und zwar in der Marx'schen Version, die nicht nur die Kategorie der Differenzialrente kennt, die aus den unterschiedlichen natürlichen Produktionsbedingungen entspringt, und die nicht preisbestimmend ist, sondern auch die Kategorie der absoluten Grundrente, die ein kapitalistisches Grundbesitzermonopol zur Voraussetzung hat, und die preisbestimmend ist. Masserrat gehört zu den wenigen Forschern, die die Bedeutung der Marx'schen Rententheorie nicht nur für die Analyse der Energie- und Rohstoff problematik erkannt, sondern darüber hinaus auch für die Analyse der internationalen Arbeitsteilung nutzbar gemacht haben.

In Teil I des Buches werden einmal die Marx'sche Rententheorie erläutert und mit ihrer Hilfe die gesellschaftlichen Bedingungen der internationalen Rohstoffproduktion und Rohstoffvermarktung analysiert, wobei es dem Autor gelingt, die gesellschaftlichen Veränderungen in den Ländern der Dritten Welt herauszuarbeiten, die u. a. auch zu der für die westliche Welt so überraschenden Ölpreiserhöhung von 1973/74 geführt haben. Gemessen am gegenwärtigen Stand der Rohstoffdiskussion ist dieser Teil der Untersuchung ausgesprochen lesenswert (und darüber hinaus im Vergleich zu anderen marxistischen Untersuchungen auch lesbar). Wenn man vielleicht auch nicht allen Thesen und Schlußfolgerungen zu folgen vermag, bieten diese Ausführungen interessante Anregungen, die den Leser zwingen, überkommene Vorstellungen zur internationalen Arbeitsteilung und Rohstoff produktion neu zu überdenken. Sehr nüchtern werden hier einige Mythen auch der marxistischen Entwicklungsländerforschung über Bord geworfen, so etwa die weitverbreitete Annahme, man könne Unterentwicklung generell als Folge einer über die internationale Handelsbeziehun- 